
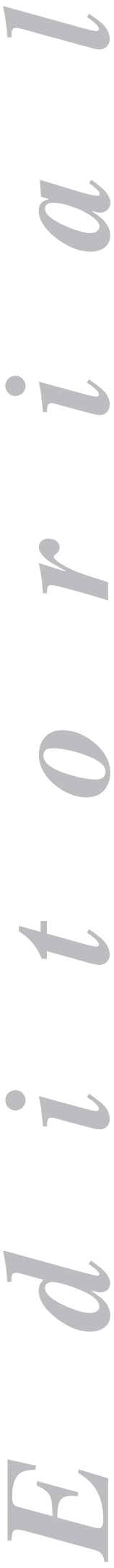

\section{Cirugía endoscópica por orificios naturales (NOTES). ¿Visión de futuro?}

Es evidente que uno de los avances médicos más importantes que han acontecido en los últimos años es la aparición de la cirugía laparóscopica (CL), no sólo por las implicaciones médicas sino también por las sociales. Su amplia incorporación, su progresión y su reproducibilidad han sido piezas fundamentales en su aceptación y probablemente no había existido un hecho tan relevante desde la aparición de la anestesia y los antibióticos.

La laparoscopia diagnóstica (LD), practicada desde hace años por gastroenterólogos, permitía valorar superficies peritoneales, hepáticas o utilizarla como soporte a la hora de efectuar biopsias hepáticas.

La realización de una colecistectomía por laparoscopia en el año 1987 por Mouret representó un nuevo concepto de la laparoscopia y una verdadera revolución en el ámbito quirúrgico. Introdujo conceptos radicalmente nuevos: por ejemplo, que el tamaño de la incisión "sí importa", ya que influye en la evolución y en el postoperatorio (íleo paralítico) y que la tecnología es fundamental para el cirujano en el desarrollo de la CL. Desde entonces, la CL ha sido utilizada con múltiples finalidades quirúrgicas y puede considerarse de elección en el tratamiento de la litiasis biliar, el tratamiento del reflujo esófago-gástrico, esplenectomía, resecciones de colon, apéndice, obesidad y en situaciones especiales en patologías del esófago, estómago, páncreas e incluso hígado $(1,2)$.

Todas estas técnicas laparoscópicas permiten disminuir el dolor, tener menor morbilidad y una estancia postoperatoria más corta con unos resultados estéticos mejores que con la cirugía abierta. Teniendo en cuenta estos aspectos, se empieza a desarrollar una serie de procesos que se engloban dentro del concepto de cirugía mínimamente invasiva.

El siguiente paso en la cirugía laparoscópica, y en un intento de disminuir la agresión, fue la reducción del tamaño de los orificios empleando trócares que han llegado a ser de tan sólo $3 \mathrm{~mm}$ (minilaparoscopia); si bien conceptualmente esto es así, los avances técnicos no han sido suficientes y los equipos actuales no permiten aplicarlo de forma sistemática.

De forma paralela se realizaba una cirugía laparoscópica que obligaba a la apertura de las vísceras, es decir, se trataba de una cirugía intraluminal (derivaciones de pseudoquistes a través del estómago, resecciones de lesiones polipoideas de estómago o colon). De esta forma se perdió el miedo a esta apertura y se rompió el concepto clásico de que la contaminación de la cavidad abdominal provocaba un claro efecto perjudicial. Esta técnica ha perdido relevancia en la actualidad, no utilizándose habitualmente.

Durante estos años, la endoscopia flexible ha evolucionado y progresado de forma geométrica, comparable a los avances que ha habido en el campo quirúrgico, ya 
que pasó de ser meramente diagnóstica a intervencionista y terapéutica, llegando hasta el momento actual (ecoendoscopia diagnóstica y terapéutica). Hoy en día es posible realizar esfinterectomías, drenajes, resecciones de lesiones de espacios endoluminales (3) o incluso derivaciones con anastomosis y crear mecanismos valvulares en el tratamiento del reflujo (4) o restricciones gástricas en la obesidad (5).

Parecía pues lógico en esta evolución de la cirugía mínimamente invasiva plantear como siguiente paso la realización de las actuaciones quirúrgicas a través de los orificios naturales (NOTES). Básicamente, consiste en el acceso a la cavidad peritoneal a través de algún orificio natural, ya sea del tubo digestivo (estómago, colon) $(6,7)$, de la vagina (8) o vejiga urinaria (9) y realizar la intervención quirúrgica mediante un endoscopio flexible.

La aplicación de esta técnica requiere una estricta colaboración entre cirujanos y endoscopistas. Parece que esto no es un problema insalvable, ya que existen situaciones en las que se ha demostrado que esta colaboración es franca, como puede ser, por ejemplo, la práctica de una colecistectomía laparoscópica en la que en el mismo acto o con anterioridad a este se realiza una papilotomía endoscópica para tratar una coledocolitiasis. Además, actualmente existen sociedades (Sociedad Catalana Médico-quirúrgica de Endoscopia Digestiva creada hace 17 años en el seno de la Academia de Ciencias Médicas de Cataluña, sociedades internacionales como SAGES, ASGE y EAES) en las que convergen cirujanos y endoscopistas-gastroenterólogos para tratar problemas afines.

Esta técnica se puede realizar ya sea asistida por laparoscopia o sin ella. Consiste en que, mediante un endoscopio flexible de $120 \mathrm{~cm}$ de longitud, de grueso calibre, con varios canales de trabajo (iluminación, aspiración e instrumental) de 3,54,2 mm de diámetro, se efectúan intervenciones tales como la colecistectomía (Marescaux, 2007), apendicetomía (Rao y Reddy, 2004), esplenectomía (10), nefrectomía, ligadura de trompas preservando la pared abdominal, incluso suturas intestinales $(11,12)$. Una problemática a tener en cuenta es la dificultad existente para conseguir una orientación espacial adecuada que permita establecer dónde se localiza la parte superior o inferior, el lado derecho o izquierdo.

El número de complicaciones asociadas a la práctica de los orificios de entrada en la cavidad abdominal a través de las vísceras es menor que el que surge cuando ello se hace a través de la pared abdominal; sin embargo, la gravedad de un posible fallo de sutura puede ser muy importante y ocasionar la pérdida de los beneficios ligados a una invasión mínima, que, por otro lado, es el objetivo de esta intervención. Uno de los campos de estudio actuales está dirigido a resolver este problema y conseguir métodos reproductibles y fiables que aseguren el cierre de los oficios practicados en la vía de entrada (13).

Es obvio que la laparoscopia representó un gran avance dentro del ámbito quirúrgico, pero la incorporación de los cirujanos a esta nueva técnica no fue lo suficientemente ordenada y, probablemente, muchos pacientes pagaron una factura desmesurada para cubrir la curva de aprendizaje del colectivo quirúrgico. Si de algo ha de servir el conocimiento de esta realidad es para que no se repita. En la aplicación del NOTES se han elaborado unas normas de incorporación que deben ser seguidas de forma estricta. Es lo que se conoce como white paper, papel blanco auspiciado por el grupo NOSCAR (14) y por la ASGE/SAGES (15). Estas normas se encuentran en continua revisión en sesiones periódicas, de las que próximamente se celebrará la tercera reunión. Los grupos que se impliquen en el tema deberán cumplir dichos criterios y deberían excluirse situaciones de "una carrera protagonística" con comunicaciones esporádicas. 


\section{Editorial}

Por otro lado, la tecnología debe progresar de forma importante con el fin de conseguir materiales que permitan lograr una buena tracción de los tejidos y su triangulación $(13,16)$. La pincería actual, por su tamaño, no permite tensionar tejidos, traccionar lesiones de gran tamaño o atrapar estructuras cuando existen procesos inflamatorios sobreañadidos.

Utilizando la metodología aplicada por NOTES, ha aparecido el término TUES (trans-umbilical endoscopic surgery), que se aplica cuando se utiliza el ombligo como puerta de entrada para el endoscopio con los canales de trabajo (17). Las dificultades para el cierre del orificio desaparecen y las posibles complicaciones de su fallo quedan mermadas. Es evidente que esta tecnología se encuentra aún en sus inicios y es difícil prever cuáles serán sus posibilidades futuras y sus indicaciones; de todas formas, no cabe duda de que su avance se encuentra estrechamente ligado a los avances técnicos y tecnológicos que influirán en otros campos de la cirugía.

La postura ante esta técnica debe ser crítica pero no escéptica y asumirla con mentalidad abierta, como siempre ha ocurrido mayoritariamente en el colectivo quirúrgico; aunque no es menos cierto, y de recuerdo no tan lejano, que en ocasiones la incorporación de nuevas técnicas causa actitudes contrarias que el tiempo termina por situar en su lugar. Así pues, debemos dejar pasar el tiempo, el cual será el verdadero juez que resolverá nuestras dudas actuales.

El grupo de Mallorca liderado por los Dres. Dolz (digestólogo) y Noguera (cirujano) ha presentado recientemente en esta revista el caso de una colecistectomía transvaginal combinada con laparoscopia y en este número comunican la realización de una resección hepática (18). Como queda reflejado en el artículo, se trata de la primera ocasión en la que se realiza una resección hepática en el ser humano por vía NOTES. El caso merece especial atención, dado que se trataba de una paciente con antecedentes de una histerectomía, lo que sin duda debería dificultar la vía de acceso vaginal por las posibles adherencias intestinales, como así fue. Se requirió la introducción de un trócar accesorio (se utilice o no posteriormente) lo que, unido a la necesidad de separar las asas intestinales de la pelvis, añadía un potencial riesgo de lesión intestinal, lo que mermaría las ventajas propias del NOTES por vía vaginal. Por otro lado, la lesión hepática es de pequeño tamaño y se encuentra localizada en el borde hepático yuxta-vesicular. Su resección podríamos incluirla en el término de pequeña resección o cuña hepática, más que de resección hepática propiamente dicha que, aunque semánticamente correcto, puede originar dudas. De forma similar a como se utiliza en las resecciones hepáticas por laparoscopia $(1,2)$, incluir términos como la localización y tamaño de la lesión puede dar una idea de la dificultad técnica de dicha resección por NOTES. Se debe agradecer a los autores de este artículo sus esfuerzos en el desarrollo de esta técnica novedosa, siguiendo una línea de investigación que supera la simple comunicación de un caso anecdótico, como queda demostrado por los artículos que este grupo ha publicado previamente en esta revista (19).

La comunidad científica está especialmente interesada en este campo, como lo demuestra el alto número de publicaciones que aparecen (20-25) y necesita conocer y contrastar los resultados de los diferentes grupos de trabajo como el de Mallorca para establecer las posibilidades reales del NOTES.

\section{E. Cugat Andorra y M. J. Varas Lorenzo ${ }^{1}$}

Servicio de Cirugía General y Digestiva. Centro Médico Teknon. Unidad de Cirugía Hepatobiliopancreática. Hospital Mútua de Terrassa. Universidad de Barcelona. ${ }^{~}$ Unidad de Ecoendoscopia. Servicio de Aparato Digestivo. Centro Médico Teknon. Barcelona 


\section{Bibliografía}

1. Cugat E, García-Domingo MI, Bretcha P, Rodríguez A, Marco C. Laparoscopia y cirugía hepática: técnica e indicaciones. Cir Esp 2004; 75 (1): 23-8.

2. Cugat E, Olsina JJ, Rotellar F, Artigas V, Suárez MA, Moreno-Sanz C, Herrera J, et al. Resultados iniciales del Registro Nacional de Cirugía Hepática por laparoscopia. Cir Esp 2005; 78 (3): 152-60.

3. Rosen MJ, Heniford BT. Endoluminal gastric surgery: The modern era of minimally invasive surgery. Surg Clin North Am 2005; 85: 989-1007.

4. Portale G, Filipi CJ, Peters JH. A current assessment of endoluminal approaches to the treatment of gastroesophageal reflux disease. Surg Innov 2004; 11: 225-34.

5. Hu B, Chung SC, Sun LC, Kawashima K, Yamamoto T, Cotton PB, et al. Transoral obesity surgery: Endoluminal gastroplasty with an endoscopic suture device. Endoscopy 2005; 37: 411-4.

6. Kalloo AN, Singh VK, Jagannath SB, et al. Flexible transgastric peritoneoscopy: A novel approach to diagnostic and therapeutic interventions in the peritoneal cavity. Gastrointest Endosc 2004; 60: 114-7.

7. Hochberger J, Lamade W. Transgastric surgery in the abdomen: The dawn of a new era? Gastrointest Endosc 2005; 62: 293-6.

8. Zorron R, Maggioni LC, Pombo L, Oliveira AL, Carvalho GL, Filgueiras M. NOTES transvaginal cholecystectomy: Preliminary clinical application. Surg Endosc 2008; 22: 542-7.

9. Gettman MT, Blute ML. Transvesical peritoneoscopy: Initial clinical evaluation of the bladder as a portal for NOTES. Mayo Clin Proc 2007; 82: 843-5.

10. Kantsevoy SV, Hu B, Jagannath SB, Vaughn CA, Beitler DM, Cheng SS, et al. Transgastric endoscopic splenectomy: Is it possible? Surg Endosc 2006; 20: 522-5.

11. Mintz Y, Horgan S, Cullen J, Falor E, Talamini MA. Dual-lumen natural orifice translumenal endoscopic surgery (NOTES): A new method for performing a safe anastomosis. Surg Endosc 2008; 22: 348-51.

12. Kantsevoy SV, Jagannath SB, Niiyama H, Chung SS, Cotton PB, Gostout CJ, et al. Endoscopic gastrojejunostomy with survival in a porcine model. Gastrointest Endosc 2005; 62: 287-92.

13. Swanström LL, Kozarek R, Pasricha PJ, Gross S, Birkett D, Park PO, et al. Development of a new access device for transgastric surgery. J Gastrointest Surg 2005; 9: 1129-36.

14. Natural Orifice Surgery Consortium for Assessment and Research (NOSCAR). Internacional Conference on Natural Orifice Translumenal Endoscopic Surgery (NOTES). Available at: http://www.noscar.org

15. Rattner D, Kalloo AN, ASGE/SAGES Working Group. ASGE/SAGES working group on Natural Orifice Transluminal Endoscopy. Gastrointest Endosc 2006; 63: 199-203.

16. Swanström LL. Desarrollo tecnológico actual de la cirugía endoscópica transluminal a través de orificios naturales. Cir Esp 2006; 80: 283-8.

17. Zhu JF. Scarless endoscopic surgery: NOTES or TUES. Surg Endosc 2007; 21: 1898-9.

18. Noguera JF, Dolz C, Cuadrado A, Olea JM, Vilella A. Transvaginal liver resection (NOTES) combined with minilaparoscopy. Rev Esp Enferm Dig 2008; 100 (7): 411-5.

19. Dolz C, Noguera JF, Martín A, Vilella A, Cuadrado A. Colecistectomía transvaginal (NOTES) combinada con minilaparoscopia. Rev Esp Enferm Dig 2007; 99 (12): 698-702.

20. Fritscher-Raven A. Transgastric endoscopy -A new fashion, a new excitement. Endoscopy 2007; $39: 161-7$.

21. Baron TH. NOTES. Br J Surg 2007; 94: 1-2.

22. Pearl JP, Ponsky JL. NOTES: Past, present and future. J Min Access Surg 2007; 3: 43-6.

23. Swain P. A justification for NOTES -Natural orificie translumenal endosurgery. Gastrointest Endosc 2007; 65: 514-6.

24. Varas MJ, Espinós JC, Abad R, Bargalló D. Endocirugía guiada por endosonografia. En: Varas MJ, editor. Ultrasonografia Endoscópica o Endosonografía. Madrid: Ed. Médica Panamericana; 2008. p. 313-20.

25. Giday SA, Magno P, Kalloo AN. NOTES: The future. Gastrointest Endosc Clin N Am 2008; 18 (2): $387-95$. 